Chris Medcraft and Melanie Schnell*

\title{
A Comparative Study of Two Bicyclic Ethers, Eucalyptol and 1,4-Cineole, by Broadband Rotational Spectroscopy
}

DOI 10.1515/zpch-2015-0643

Received June 5, 2015; accepted August 5, 2015

Abstract: The rotational spectra of the two structurally related molecules, 1,4cineole and 1,8-cineole (eucalyptol), were measured between $2-8.5 \mathrm{GHz}$ with chirped pulse Fourier transform microwave spectroscopy. The structures of these two molecules only differ in the connectivity of an ether functional group. This results in a significant change in the three dimensional structure of the molecule and consequently large differences in the rotational spectra. Only one conformer of each molecule was detected in the molecular jet and no line splittings due to internal rotations were detected. A substitution structure $\left(r_{s}\right)$ was determined for eucalyptol and benchmarked to quantum chemical structures allowing for a comparison of eucalyptol and 1,4-cineole.

Keywords: Microwave Spectroscopy, Chirped-pulse Fourier Transform Rotational Spectroscopy, Atmospheric Chemistry.

\section{Introduction}

Terpenes are a diverse class of Biogenic Volatile Organic Compounds (BVOCs) that are produced via the addition of building block units of isoprene (2-methyl1,3-butadiene). Monoterpenoids contain two such units and an additional functional group (e.g. alcohol or ketone) and can take on a great variety of struc-

\footnotetext{
*Corresponding author: Melanie Schnell, Max-Planck-Institut für Struktur und Dynamik der Materie, Luruper Chaussee 149, D-22761 Hamburg, Germany; and Center for Free-Electron Laser Science, Luruper Chaussee 149, D-22761 Hamburg, Germany, e-mail: melanie.schnell@mpsd.mpg.de

Chris Medcraft: Max-Planck-Institut für Struktur und Dynamik der Materie, Luruper Chaussee 149, D-22761 Hamburg, Germany; and Center for Free-Electron Laser Science, Luruper Chaussee 149, D-22761 Hamburg, Germany
} 

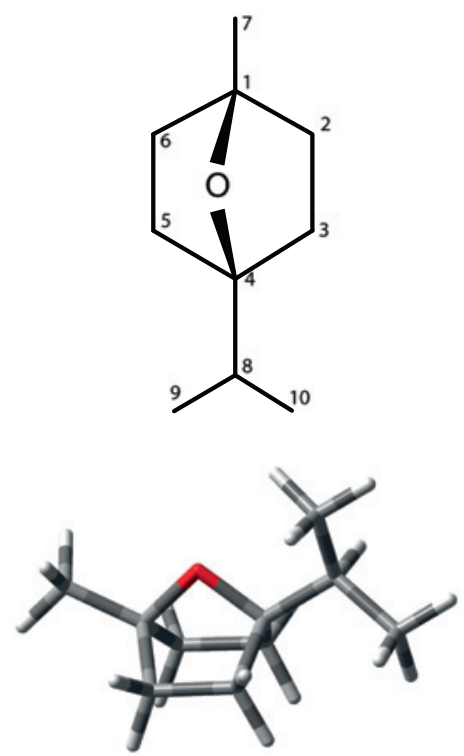
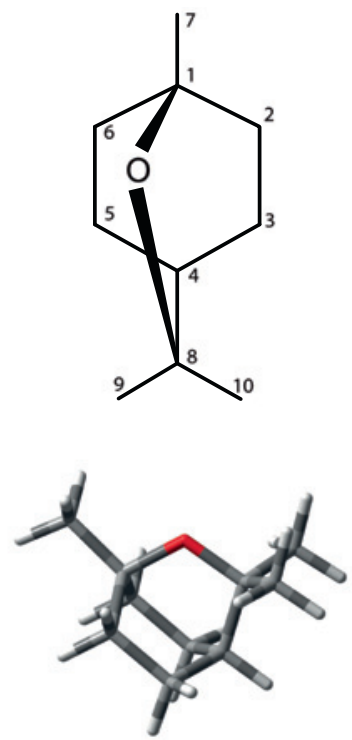

Figure 1: Scheme of the molecular structures as well as a three-dimensional view of 1,4-cineole (left) and 1,8-cineole (eucalyptol, right). Both molecules are orientated with their methyl groups to the left and isopropyl groups on the right. The cyclohexane ring is in the boat conformer in both molecules, bridged by the ether linkage.

tures based on the connectivity and degree of saturation. Emissions of these types of molecules play an important role in atmospheric chemistry via reactions with $\mathrm{O}_{3}, \mathrm{OH}$ or $\mathrm{NO}_{3}$ [1]. They can also have significant insecticidal [2, 3], herbicidal [4], anti-microbial [5, 6], and medicinal properties [7, 8]. A fundamental knowledge of the physical chemistry of these molecules is vital in understanding the complex interactions in atmospheric and biological environments. In this work we report the microwave spectra of two structurally related monoterpenoids: 1,8-cineole $\left(\mathrm{C}_{10} \mathrm{H}_{18} \mathrm{O}\right.$, 1,3,3-trimethyl-2-oxabicyclo[2.2.2]octane) and 1,4-cineole $\left(\mathrm{C}_{10} \mathrm{H}_{18} \mathrm{O}\right.$, 1-isopropyl-4-methyl-7-oxabicyclo[2.2.1] heptane). The structures of 1,8cineole, hereafter eucalyptol (Figure 1 right), and 1,4-cineole are atypical of most monoterpenoids as they are fully saturated, bicyclic ethers. A three dimensional representation of both molecules is shown in the lower part of Figure 1.

Eucalyptol is widely used in the cosmetics industry and has known herbicidal [9] and insecticidal properties [10]. Due to the lack of more reactive functional groups the atmospheric lifetime of eucalyptol is on the order of one day [11], significantly longer than most monoterpenes with typical lifetimes on the order of hours [12]. However, the atmospheric lifetime of eucalyptol is significantly shorter than that of many ethers cf. dimethyl ether (5.1 days) [13], diethyl ether 
(12 days) [14] and ethyl tert-butyl ether (40 days) [14] whilst being longer than monocyclic ethers like tetrahydrofuran (6 hours) [15]. It is thus interesting to study if the atmospheric lifetime of eucalytol can be explained by the molecular structure, which is one aim of the present study.

Despite the lower reactivity, when compared to other monoterpenes, sufficiently large quantities of eucalyptol are produced in eucalyptus forests that it can be a major factor in local atmospheric chemistry and particle formation [16]. The primary reaction pathway of atmospheric eucalyptol is via hydrogen abstraction by $\mathrm{OH}$ radicals [11] which leads to an alkoxy radical which is then oxidised by $\mathrm{O}_{2}$ [12]. A detailed mechanism was proposed by linuma and co-workers which leads to highly oxygenated species (diaterebic acid acetate and diaterpenylic acid acetate). These species are proposed to be important in secondary organic aerosol formation [17]. This mechanism involves two ring opening reactions which, interestingly, does not include the breaking of the $\mathrm{C}-\mathrm{O}-\mathrm{C}$ linkage which one might assume to be strained in the bicyclic nature of eucalyptol and susceptible to cleavage. A detailed knowledge of the equilibrium structure may help elucidate the fundamental reasons for this mechanism and which bonds are most likely to break.

Whilst 1,4-cineole (Figure 1 left) is only a minor component of some essential oils [4, 18], and thus less relevant to atmospheric chemistry, it has been shown to have phototoxic properties [9], and is used as the basis for the herbicide cinmethylin. [19] Intriguingly the biological mode of action of 1,4-cineole appears to be different to that of eucalyptol despite the similarity in structure [4]. This difference in biochemistry makes for an interesting comparison for structural determination methods such as microwave spectroscopy.

Broadband microwave spectroscopy is a versatile and powerful tool to investigate the three dimensional structure of molecules in the gas phase. This was impressively shown for strawberry aldehyde [20], large water complexes [21-23] and cinnamaldehyde [24]. Recently a number of monoterpenoids have been investigated via microwave spectroscopy. The acyclic linalool [25] was found to have a great amount of conformer flexibility, however, due to the low barriers of rotation only one conformer was observed in the cold conditions of the molecular jet.

Monocyclic monoterpenes often have a structure consisting of a six membered carbon ring with a methyl and isopropyl substituents. This basic structure can lead to a large variety of molecular structures which have a wide range of characteristic odours that are exploited by the cosmetic industry. The relationship between odour and molecular structure is not well understood [26] and therefore it is an intriguing topic to study. This is one of the reasons that many of these compounds have been studied by microwave spectroscopy including limonene [27], perillaldehyde [28, 29], carvone [27], and estragole [30]. Our group has recently 
investigated carvacrole, thymol [31] menthol, menthone, isomenthone [32] and carvomenthenol [33] and with the present study we extend this series to bicyclic monoterpenes.

Many bicyclic monoterpenes retain the monocyclic basis of a six-membered carbon ring and in addition contain a linkage between two carbons to form a second ring. Intuitively this can lead to abnormal bonding geometries and can limit the number of possible conformations. Early work on six bicyclic monoterpenes was performed in 1978 [34]. These molecules were all based on the general structure of 3-bicyclo[3.1.0]hexanone, which consists of a six-membered ring with a bond between carbons 1 and 5 forming a secondary three-membered ring. By using geometric assumptions from a detailed investigation of bicyclo[3.1.0]hexanone [35] this low resolution study could identify the general conformational structures of each of the six molecules. For $\alpha$ - and $\beta$-pinene a carbon atom links the 1 and 3 positions of a six-membered ring to form a secondary four-membered ring, they differ in the position of a $\mathrm{C}-\mathrm{C}$ double bond. Only one conformer was observed and no splitting of lines due to internal rotation of the methyl groups was seen [36]. Similar results were seen in nopinone [36] and verbenone [37] which are ketones with similar carbon backbone structures to pinene.

Camphor, which has an analogous 1,4 carbon linkage across a cyclohexane ring, was studied by Kisiel et al. [38] via microwave spectroscopy and a heavy atom structure was reported. The bicyclic hydrocarbons norbornane [39] and norbornene [40] were studied via Stark modulated microwave spectroscopy. These bicyclic compounds are also interesting from a fundamental physical chemistry point of view as the bonds are often strained into unusual geometries. The conformational flexibility (or lack thereof) is particularly interesting for terpenes as it relates to the structure-function relationship and how compounds with similar structures can have vastly different chemical or biochemical properties.

\section{Experimental}

The spectra were recorded on the COMPACT broadband microwave spectrometer in Hamburg, the technical details of which have been given elsewhere [41]. The samples of eucalyptol ( $99 \%$ stated purity) and 1,4-cineole (85\% purity) were purchased from Sigma-Aldrich and used without further purification. Both samples are liquids at room temperature and were placed into a sample reservoir immediately behind a series 9 general valve. The reservoir and valve were heated to obtain sufficient vapour pressure, $120^{\circ} \mathrm{C}$ for eucalyptol and $30^{\circ} \mathrm{C}$ for 1,4-cineole. The molecules were expanded into the vacuum chamber by a backing pressure of 
neon at 2 bar. A microwave chirp spanning $2-8.5 \mathrm{GHz}$ in $1 \mu$ s was used to excite the molecules and the molecular response, in the form of a free induction decay in the time domain, was then recorded directly on a fast oscilloscope.

For the eucalyptol experiments a total of 1 million free induction decays of $50 \mu$ s length were coadded and Fourier transformed using a Kaiser-Bessel window function to give the frequency spectrum with a point to point resolution of approximately $20 \mathrm{kHz}$. In the 1,4-cineole experiment approximately 500000 FIDs were likewise coadded and Fourier transformed to give the spectrum.

\section{Results}

\subsection{Eucalyptol}

The only appreciable dipole moment component of eucalyptol lies along the $b$ inertial axis (see Table 1) and thus a pure b-type spectrum is observed. Within the frequency range of the spectrometer the spectrum of eucalyptol contains a number of Q-branches $\left(\Delta J=0, \Delta K_{a}=+1, \Delta K_{c}=-1\right)$ that were readily identified via their distinctive pattern (see top right panel of Figure 2). A total of 217 Q-branch lines were assigned over six branches from $K_{a}=2$ to $K_{a}=7$. A comparatively smaller number (22) of P-branch lines were identified, however these were invaluable in decoupling the three rotational constants $(A, B, C)$ from each other in the fit to a rigid rotor Hamiltonian (vide infra). This initial assignment of the 239 transitions was performed in the PGOPHER program suite [42]. The list of transitions and experimental frequencies were then fit to Watson's S-reduced Hamiltonian [43] as implemented in SPFIT [44].

Due to the high proportion of Q-branch lines the $A, B$ and $C$ rotational constants were highly intercorrelated in the initial fit. In a fit that only includes Qbranch lines the $A, B$ and $C$ constants are perfectly coupled and the fit does not converge. The inclusion of the $D_{J}$ centrifugal distortion term makes this even worse as it, too, is correlated to $A, B$ and $C$. The inclusion of P-branch lines to the fit does improve the situation, however, intercorrelation factors of $A, B$, and $C$ were still all above 0.97 . As a means to reduce these interdependencies, linear combinations of $B$ and $C$ were fit, i.e. $\frac{B+C}{2}$ and $\frac{B-C}{4}$. Whilst $A$ is still coupled to $B+C$ it was decoupled from $B-C$. In this way all quartic centrifugal distortion constants could be successfully fit. This coupling of constants is likely caused by the small number of $\Delta J=-1$ transitions and the small quantum number range $(J=1-5)$ of them. Measurement of higher $J$ P-branch transitions could further de- 


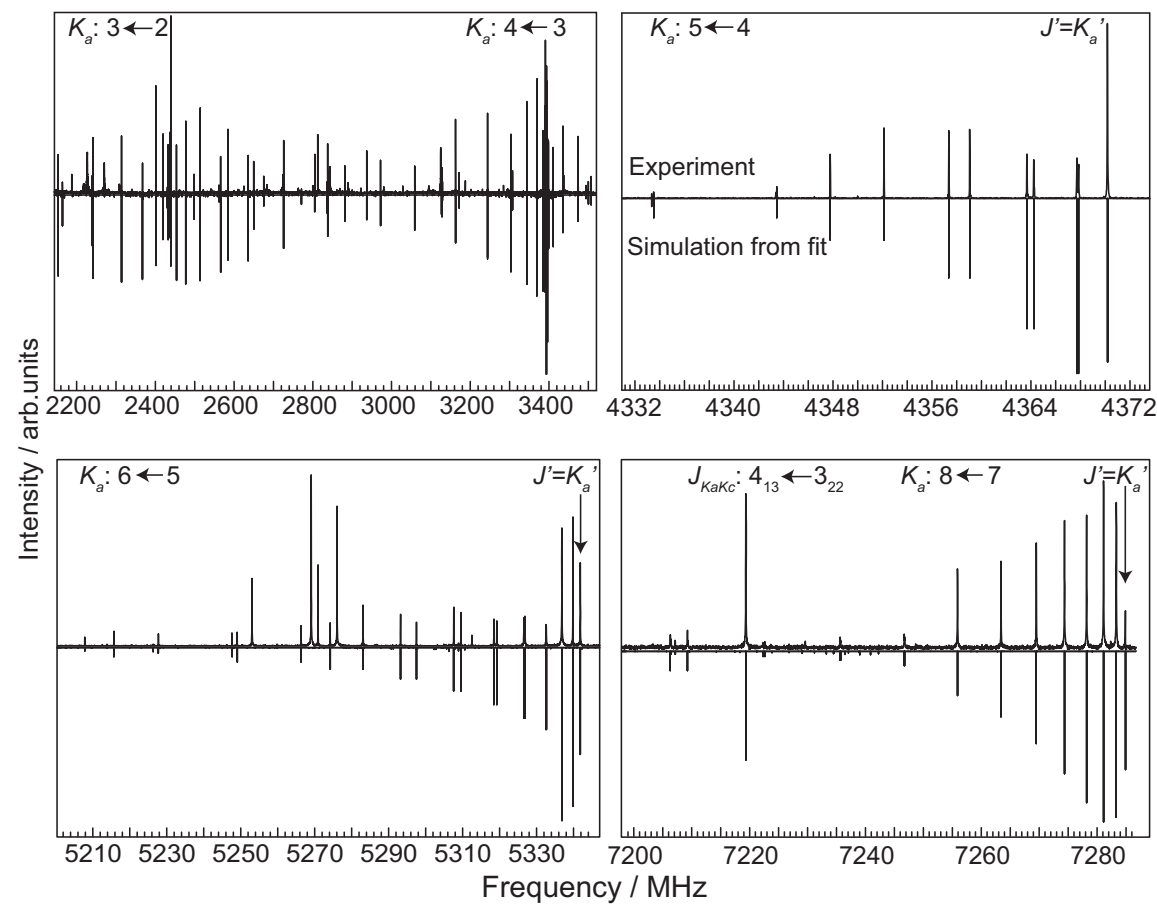

Figure 2: Sections of the broadband microwave spectrum of eucalyptol, highlighting the Q-branch structures. The lines marked as $J^{\prime}=K_{a}^{\prime}$ are the start of the Q-branches, with $J^{\prime}$ increasing with decreasing frequency, whilst $K_{a}^{\prime}$ remains the same. The splitting that is seen in some areas (e.g. at $4364 \mathrm{MHz}$ ) is due to the breaking of the $K_{c}$ even/odd degeneracy. The lines around $5270 \mathrm{MHz}$ in the lower left panel are from residual acetone.

couple the constants, unfortunately the bulk of these transition lie outside of the spectrometer's frequency range.

A summary of this fit is presented in Table 1 alongside results of a number of quantum chemical calculations performed with the Gaussian09 program suite [45]. A full list of assigned transitions can be found in the supplementary material. The best performing method/basis set combination was B3LYP/cc-pVTZ, although all approaches predict rotational constants to within $15 \mathrm{MHz}$. The structure obtained from the B3LYP/cc-pVTZ calculation was subsequently used for a vibrational calculation to compute centrifugal distortion constants. While no line splittings were observed in the spectrum there is the possibility that the three methyl groups (C7, C9, and C10 in Figure 1) could act as internal rotors and cause such a splitting. Relaxed potential energy scans (B3LYP/cc-pVDZ) of each methyl group showed barrier heights of approximately $12 \mathrm{~kJ} / \mathrm{mol}\left(1000 \mathrm{~cm}^{-1}\right)$ which sug- 
Table 1: Experimental and ab initio rotational constants of eucalyptol.

\begin{tabular}{lrrrr}
\hline & Fit & B3LYP/cc-pVTZ & M062X/cc-pVTZ & MP2/cc-pVTZ \\
\hline$A / \mathrm{MHz}$ & $1545.88790(43)^{\mathrm{a}}$ & 1546.77 & 1537.35 & 1560.71 \\
$\frac{B+C}{2} / \mathrm{MHz}$ & $1059.70055(43)$ & 1053.98 & 1069.72 & 1071.14 \\
$\frac{B-C}{4} / \mathrm{MHz}$ & $10.383693(10)$ & 10.39 & 10.31 & 10.55 \\
$B / \mathrm{MHz}$ & 1080.467936 & 1074.76 & 1075.85 & 1092.253 \\
$C / \mathrm{MHz}$ & 1038.933164 & 1033.19 & 1033.79 & 1050.032 \\
$D_{J} / \mathrm{kHz}$ & $0.024(12)$ & 0.0295 & & \\
$D_{J K} / \mathrm{kHz}$ & $0.01092(28)$ & 0.0390 & & \\
$D_{K} / \mathrm{kHz}$ & $-0.0354(14)$ & -0.0121 & & \\
$d_{1} / \mathrm{Hz}$ & $-1.321(29)$ & -1.32 & & \\
$d_{2} / \mathrm{Hz}$ & $1.6695(79)$ & 1.87 & & \\
$\sigma / \mathrm{kHz}$ & 5.5 & & & \\
$N$ & 239 & & & \\
$\mathrm{Max} J / K_{a} / K_{c}$ & $25 / 8 / 21$ & & & \\
$\mu_{a} / \mu_{b} / \mu_{c}$ & & $-0.05 / 1.39 / 0.00$ & $-0.05 /-1.41 / 0.00$ & $-0.03 / 1.62 / 0.00$ \\
\hline
\end{tabular}

${ }^{a}$ Numbers in parenthesis are standard errors in the units of the least significant digit of the constant.

gests our experiment would not be sensitive to any such splitting at this resolution $(\simeq 20 \mathrm{kHz})[25]$.

Further inspection of the spectrum revealed lines from the singly substituted ${ }^{13} \mathrm{C}$ isotopologues in natural abundance. The quantum chemical calculations suggested that eucalyptol possesses a plane of symmetry through $\mathrm{C} 7, \mathrm{C} 1, \mathrm{C} 4$, and $\mathrm{C} 8$ in Figure 1 ( $C_{s}$ point group), which results in three pairs of equivalent carbon atoms (C2 \& C6, C3 \& C5, C9 \& C10). This was confirmed by the presence of only seven unique singly substituted ${ }^{13} \mathrm{C}$ isotopologues in the spectrum. Between 6 and 17 transitions were assigned to each isotopologue and rotational constants were fit to Watson's S-reduced Hamiltonian. The results of these fits are shown in Table 2. The quartic centrifugal distortion constants were fixed to those of the parent species (see Table 1). Since most of the observed ${ }^{13} \mathrm{C}$ lines were P-branch $(\Delta J=-1)$, the rotational constants could be fit independently with little intercorrelations.

The rotational constants were then used to calculate atomic positions using Kraitchmann's equations [46] as implemented in Kisiel's KRA program [47]. A few of the atomic coordinates were imaginary as they were close to a molecular axis. These coordinates were set zero and the magnitude of the imaginary number was used as the uncertainty of that coordinate, see Table 3. Selected bond lengths and angles calculated from the substitution coordinates are shown in Table 4 along with calculated parameters. 
Table 2: Summary of the fits of the seven singly substituted ${ }^{13} \mathrm{C}$ isotopologues of eucalyptol. Centrifugal distortion constants are fixed to the values of the parent species shown in Table 1.

\begin{tabular}{lrrrrr}
\hline & $A / \mathrm{MHz}$ & $B / \mathrm{MHz}$ & $\mathrm{C} / \mathrm{MHz}$ & $N$ & $\sigma / \mathrm{kHz}$ \\
\hline C1 & $1545.24021(99)$ & $1077.1091(16)$ & $1035.5123(14)$ & 7 & 7.3 \\
C2/C6 & $1537.47052(54)$ & $1073.34699(51)$ & $1035.06347(60)$ & 17 & 8.4 \\
C3/C5 & $1528.81221(64)$ & $1077.3471(10)$ & $1034.0594(10)$ & 16 & 8.3 \\
C4 & $1540.05252(53)$ & $1079.00480(78)$ & $1034.71226(71)$ & 6 & 3.5 \\
C7 & $1536.83226(54)$ & $1067.91940(81)$ & $1023.31040(96)$ & 8 & 3.9 \\
C8 & $1545.3849(21)$ & $1077.1451(17)$ & $1035.6072(17)$ & 6 & 7.3 \\
C9/C10 & $1536.37159(62)$ & $1067.79327(90)$ & $1029.08190(93)$ & 15 & 8.2 \\
\hline
\end{tabular}

Table 3: Substitution coordinates of carbon atoms obtained from Kraitchman analysis.

\begin{tabular}{lrrr}
\hline & $a(\AA)$ & $b(\AA)$ & $c(\AA)$ \\
\hline$C(1)$ & $1.2114(13)$ & $-0.3804(40)$ & $0.000(76)$ \\
$C(2)$ & $1.2462(12)$ & $0.4850(31)$ & $1.2563(12)$ \\
$C(3)$ & $0.00(43)$ & $1.4611(10)$ & $1.2563(12)$ \\
$C(4)$ & $-0.8279(20)$ & $1.1444(15)$ & $0.0000(69)$ \\
$C(5)$ & $0.000(43)$ & $1.4611(10)$ & $-1.2563(12)$ \\
$C(6)$ & $1.2462(12)$ & $0.4850(31)$ & $1.2563(12)$ \\
$C(7)$ & $2.33222(66)$ & $-1.4174(11)$ & $0.0000(88)$ \\
$C(8)$ & $-1.2054(13)$ & $-0.3383(45)$ & $0.000(81)$ \\
$C(9)$ & $-2.01269(75)$ & $-0.7331(21)$ & $1.2441(12)$ \\
$C(10)$ & $-2.01269(75)$ & $-0.7331(21)$ & $-1.2441(12)$ \\
\hline
\end{tabular}

A least squares fit of the rotational constants to the structural parameters was attempted ( $r_{0}$ and $r_{m}$ structures) however the fit would not converge unless an unreasonable number of the parameters were fixed. This is perhaps due to the bond lengths and angles being highly correlated in the bicyclic structure, i.e. the length of one bond is highly dependent on the lengths and angles of neighbouring bonds. The ${ }^{18} \mathrm{O}$ isotopologue could not be observed in natural abundance $(\simeq 0.2 \%)$ with the present signal-to-noise ratio and may be useful in calculating a $r_{m}$ structure. The method for determining $r_{m}$ structures models the vibrational contributions to the zero point moments of inertia and generally give results that are closer to the equilibrium structure than the substitution method [48]. It is therefore desirable to obtain an $r_{m}$ structure when possible. It is worth noting here that in the similarly structured molecule camphor, the $r_{m}^{(1)}$ bond lengths differed, on average, by less than $0.006 \AA$ from the $r_{s}$ bond lengths [38]. This is less than most of the uncertainties in the $r_{s}$ presented here. 
Table 4: Structural parameters of eucalyptol, 1,4-cineole and simple ethers.

\begin{tabular}{|c|c|c|c|c|c|}
\hline \multirow[t]{2}{*}{ Parameter } & \multicolumn{2}{|c|}{ eucalyptol } & \multirow{2}{*}{$\begin{array}{r}\text { 1,4-cineole } \\
\text { B3LYP/cc-pVTZ }\end{array}$} & \multirow{2}{*}{$\begin{array}{r}\text { dimethyl ether } \\
\qquad\left(r_{s}\right) \text { [51] }\end{array}$} & \multirow{2}{*}{$\begin{array}{r}\text { diethyl ether } \\
\left(r_{s}\right) \text { [52] }\end{array}$} \\
\hline & $\left(r_{s}\right)$ & B3LYP/cc-pVTZ & & & \\
\hline$r\left(\mathrm{C}_{1} \mathrm{C}_{2}\right) / \AA$ & $1.527(62)$ & 1.542 & 1.541 & & \\
\hline$r\left(\mathrm{C}_{1} \mathrm{C}_{7}\right) / \AA$ & $1.527(3)$ & 1.523 & 1.509 & & \\
\hline$r\left(\mathrm{C}_{2} \mathrm{C}_{3}\right) / \AA$ & $1.550(33)$ & 1.553 & 1.512 & & \\
\hline$r\left(\mathrm{C}_{3} \mathrm{C}_{4}\right) / \AA$ & $1.545(24)$ & 1.542 & 1.557 & & \\
\hline$r\left(C_{4} C_{8}\right) / \AA$ & $1.530(5)$ & 1.553 & 1.524 & & \\
\hline$r\left(\mathrm{C}_{8} \mathrm{C}_{9}\right) / \AA$ & $1.535(65)$ & 1.554 & 1.533 & & \\
\hline$r\left(\mathrm{C}_{1} \mathrm{O}\right) / \AA$ & - & 1.444 & 1.442 & $1.410(3)$ & $1.408(2)$ \\
\hline$r\left(\mathrm{C}_{8} \mathrm{O}\right) / \AA$ & - & 1.455 & 1.446 & & \\
\hline$\angle\left(\mathrm{C}_{1} \mathrm{O} \mathrm{C}_{8}\right) /^{\circ}$ & - & 115.1 & 98.4 & 111.72(33) & $112.42(27)$ \\
\hline$\angle\left(\mathrm{C}_{4} \mathrm{C}_{3} \mathrm{C}_{2}\right) /^{\circ}$ & 108.6(5) & 108.65 & 101.68 & & \\
\hline$\angle\left(\mathrm{C}_{3} \mathrm{C}_{2} \mathrm{C}_{1}\right) /^{\circ}$ & $109.2(10)$ & 109.45 & 101.83 & & \\
\hline$\angle\left(\mathrm{C}_{2} \mathrm{C}_{1} \mathrm{C}_{6}\right) /^{\circ}$ & $110.8(3)$ & 109.64 & 108.83 & & \\
\hline$\angle\left(\mathrm{C}_{2} \mathrm{C}_{1} \mathrm{C}_{7}\right) /^{\circ}$ & $111.6(35)$ & 112.20 & 116.14 & & \\
\hline$\angle\left(\mathrm{C}_{3} \mathrm{C}_{4} \mathrm{C}_{8}\right) /^{\circ}$ & $109.7(26)$ & 109.91 & 117.15 & & \\
\hline$\angle\left(\mathrm{C}_{9} \mathrm{C}_{8} \mathrm{C}_{10}\right) /^{\circ}$ & $108.3(2)$ & 108.58 & 111.43 & & \\
\hline
\end{tabular}

However, the substitution structure reveals that despite the bicyclic arrangement the carbon-carbon bond lengths and angles are all surprisingly close to the "ideal” tetrahedral cases $\left(1.54 \AA\right.$ and $\left.109.5^{\circ}\right)$. Since these structural parameters are in good agreement with the $a b$ initio values it can be reasonably assumed that the $a b$ initio values of the parameters that could not be determined, namely the $\mathrm{C}-\mathrm{O}$ bond lengths and $\mathrm{C}-\mathrm{O}-\mathrm{C}$ angle, are also close to experiment. With this assumption in mind the $\mathrm{C}-\mathrm{O}$ bond lengths are found to be longer and the $\mathrm{C}-\mathrm{O}-\mathrm{C}$ bond angle is larger for eucalyptol than for dimethyl ether (DME) or diethyl ether (DEE), see Table 4. The reaction mechanisms of ethers with $\mathrm{OH}$ radicals, generally, do not involve the cleavage of the $\mathrm{C}-\mathrm{O}$ bond $[14,49]$. The longer ether bond in eucalyptol may suggest that it is more susceptible to cleavage than smaller, acyclic ethers.

\subsection{1,4-cineole}

For 1,4-cineole a total of 53 transitions ( 9 a-type, 26 b-type and 18 c-type) up to $J=9$ were fit to Watson's S-reduced Hamiltonian; the results are shown in Table 5, a full line list is in the supplementary material. Only the $D_{K}$ and $D_{J}$ centrifugal distortion constants could be fit, the $D_{J K}$ constant was significantly smaller in 
magnitude and was poorly defined. Its inclusion did not significantly decrease the standard deviation and therefore it was excluded from the fit.

The structural parameters obtained from a B3LYP/cc-pVTZ basis set are shown in Table 4 alongside the comparable parameters for eucalyptol. This method/basis set combination was found to reproduce the experimental rotational and structural parameters of eucalyptol well. It also reproduces the rotational constants of 1,4-cineole well (Table 5). It is therefore reasonable to assume that the calculated structural parameters for 1,4-cineole are approximately as accurate as those of eucalyptol. In this way it can be seen that the $\mathrm{C}-\mathrm{O}-\mathrm{C}$ bond angle is significantly smaller in the case of 1,4-cineole and many of the $\mathrm{C}-\mathrm{C}-\mathrm{C}$ bond angles deviate significantly from the ideal tetrahedral angles. This suggests that 1,4-cineole is more strained than eucalyptol which is expected due to the lower number of carbon atoms between the bridging ether linkage.

Although only one conformer was detected in the spectrum, the different connectivity of the ether group in 1,4-cineole, when compared to eucalyptol, allows for the rotation of the isopropyl group and thus permits the possibility of a number of different conformers. A relaxed potential energy scan using the B3LYP density functional and Dunning's double zeta basis set (cc-pVDZ) [50] of the H-C8-C4$\mathrm{O}$ dihedral angle showed three minima. The two lowest energy conformers are enantiomers of each other and therefore have identical rotational constants which correspond well to the experimental constants (Table 5). The higher energy conformer, as in eucalyptol, has a plane of symmetry through the ether moiety, this conformer was not detected in the spectrum.

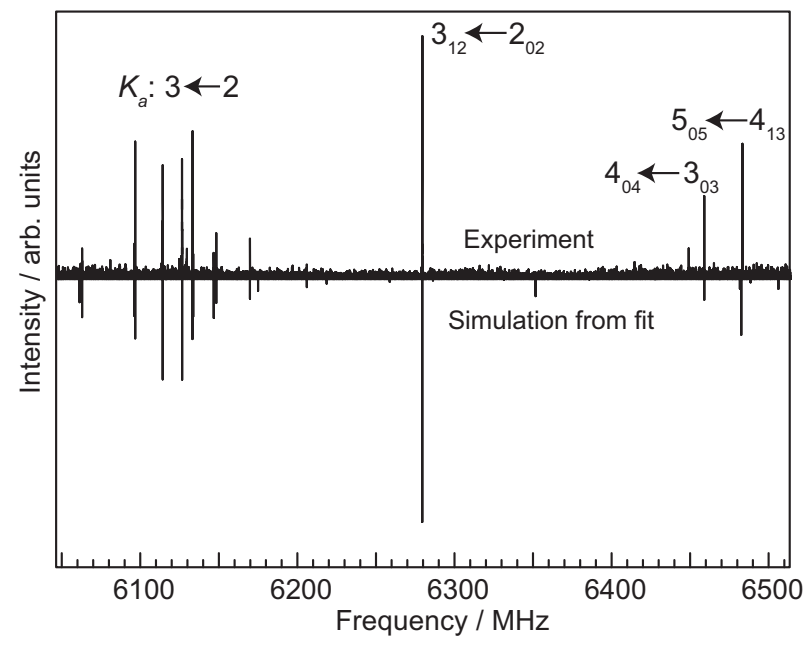

Figure 3: A portion of the broadband rotational spectrum of 1,4-cineole showing the $K_{a}: 3 \leftarrow 2$ Q-branch (left) and a number of P-branch lines. 
Table 5: Rotational constants of 1,4-cineole.

\begin{tabular}{lrr}
\hline Constant & Fitted value & B3LYP/cc-pVTZ \\
\hline$A / \mathrm{MHz}$ & $2035.46394(87)$ & 2034.66 \\
$B / \mathrm{MHz}$ & $842.27389(55)$ & 837.71 \\
$C / \mathrm{MHz}$ & $778.63056(44)$ & 774.51 \\
$D_{J} / \mathrm{kHz}$ & $0.0133(130)$ & 0.0212 \\
$D_{K} / \mathrm{kHz}$ & $-0.224(84)$ & -0.116 \\
$N$ & 53 & \\
$\sigma / \mathrm{kHz}$ & 8.2 & \\
\hline
\end{tabular}

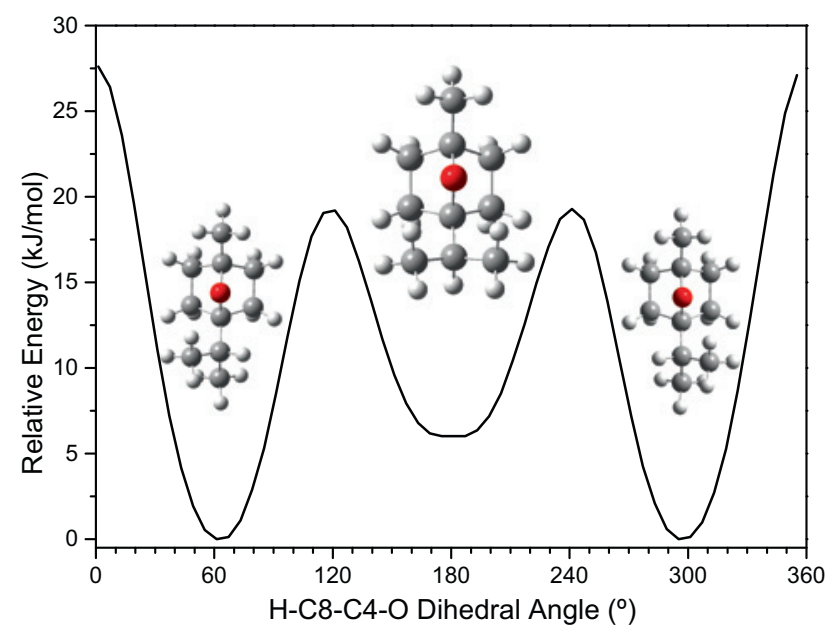

Figure 4: Relaxed potential energy scan of rotation of the isopropyl group in 1,4-cineole at the B3LYP/cc-pVDZ level.

\section{Summary}

In summary, the rotational constants for two structurally related monoterpenoids, eucalyptol and 1,4-cineole, were determined through broadband Fourier transform microwave spectroscopy. For eucalyptol the rotational constants for all seven singly substituted ${ }^{13} \mathrm{C}$ isotopologues were determined and used to determine a substitution $\left(r_{s}\right)$ structure for the vibronic ground state. For a bicyclic molecule the structural parameters showed remarkably few deviations from the ideal tetrahedral values leading to a relatively unstrained structure. Only the ether bond lengths deviate from ideal values and are not proposed to be (directly) involved in 
the atmospheric reaction mechanism [17]. This suggests that the relatively higher atmospheric reactivity, when compared to other ethers, is not due to the straining or weakening of bonds within the bicyclic structure. Benchmarked density functional theory calculations show that 1,4-cineole has a significantly more strained geometry than eucalpytol particularly with a smaller ether bond angle $\left(98^{\circ}\right)$ compared to eucalyptol $\left(115^{\circ}\right)$.

Acknowledgement: C. M. acknowledges the use of the GWDG computer cluster. M. S. acknowledges funding by the Fonds der Chemischen via a Dozentenstipendium. This work has also been supported by the excellence cluster "The Hamburg Centre for Ultrafast Imaging - Structure, Dynamics and Control of Matter at the Atomic Scale" of the Deutsche Forschungsgemeinschaft.

\section{References}

1. J. Kesselmeier and M. Staudt, J. Atmos. Chem. 33 (1999) 23.

2. H. T. Prates, J. P. Santos, J. M. Waquil, J. D. Fabris, A. B. Oliveira, and J. E. Foster, J. Stored Prod. Res. 34 (1998) 243.

3. V. Sfara, E. N. Zerba, and R. A. Alzogaray, J. Med. Entomol. 46 (2009) 511.

4. J. G. Romagni, S. N. Allen, and F. E. Dayan, J. Chem. Ecol. 26 (2000) 303.

5. W.-X. Du, C. W. Olsen, R. J. Avena-Bustillos, T. H. McHugh, C. E. Levin, and M. Friedman, J. Agr. Food Chem. 56 (2008) 3082.

6. J. Xu, F. Zhou, B.-P. Ji, R.-S. Pei, and N. Xu, Lett. Appl. Microbiol. 47 (2008) 174.

7. F. A. Santos and V. S. N. Rao, Phytother. Res. 14 (2000) 240.

8. A. Astani, J. Reichling, and P. Schnitzler, Phytother. Res. 24 (2010) 673.

9. S. F. Vaughn and G. F. Spencer, Weed Science 41 (1993) 114.

10. M. V. Maciel, S. M. Morais, C. M. L. Bevilaqua, R. A. Silva, R. S. Barros, R. N. Sousa, L. C. Sousa, E. S. Brito, and M. A. Souza-Neto, Vet. Parasitol. 167 (2010) 1.

11. S. B. Corchnoy and R. Atkinson, Environ. Sci. Technol. 24 (1990) 1497.

12. R. Atkinson and J. Arey, Atmos. Environ. 37 (2003) 197.

13. D. A. Good, J. S. Francisco, A. K. Jain, and D. J. Wuebbles, J. Geophys. Res.-Atmos. 103 (1998) 28181.

14. T. J. Wallington and S. M. Japar, Environ. Sci. Technol. 25 (1991) 410.

15. B. Cabanas, M. T. Baeza, S. Salgado, P. Martin, R. Taccone, and E. Martinez, J. Phys. Chem. A 108 (2004) 10818.

16. C. He, F. Murray, and T. Lyons, Atmos. Environ. 34 (2000) 645.

17. Y. linuma, O. Boege, M. Keywood, T. Gnauk, and H. Herrmann, Environ. Sci. Technol. 43 (2009) 280.

18. J. J. Brophy, N. W. Davies, I. A. Southwell, I. A. Stiff, and L. R. Williams, J. Agr. Food Chem. 37 (1989) 1330.

19. S. O. Duke, F. E. Dayan, J. G. Romagni, and A. M. Rimando, Weed Res. 40 (2000) 99. 
20. S. T. Shipman, J. L. Neill, R. D. Suenram, M. T. Muckle, and B. H. Pate, J. Phys. Chem. Lett. 2 (2011) 443.

21. C. Peréz, M. T. Muckle, D. P. Zaleski, N. A. Seifert, B. Temelso, G. C. Shields, Z. Kisiel, and B. H. Pate, Science 336 (2012) 897.

22. C. Peréz, S. Lobsiger, N. A. Seifert, D. P. Zaleski, B. Temelso, G. C. Shields, Z. Kisiel, and B. H. Pate, Chem. Phys. Lett. 571 (2013) 1.

23. C. Peréz, D. P. Zaleski, N. A. Seifert, B. Temelso, G. C. Shields, Z. Kisiel, and B. H. Pate, Angew. Chem. 126 (2014) 14596.

24. S. Zinn, T. Betz, C. Medcraft, and M. Schnell, Phys. Chem. Chem. Phys. (2015), DOI $10.1039 / \mathrm{C} 5 \mathrm{CP} 02582 \mathrm{~F}$.

25. H. V. L. Nguyen, H. Mouhib, S. Klahm, W. Stahl, and I. Kleiner, Phys. Chem. Chem. Phys. 15 (2013) 10012.

26. J. C. Brookes, A. P. Horsfield, and A. M Stoneham, J. R. Soc. Interface 6 (2009) 75.

27. J. R. Aviles Moreno, T. R. Huet, and J. J. Lopez Gonzalez, Struct. Chem. 24 (2013) 1163.

28. J. R. Aviles Moreno, F. P. Urena, J. J. Lopez Gonzalez, and T. R. Huet, Chem. Phys. Lett. 473 (2009) 17.

29. T. R. Huet, J. R. Aviles Moreno, O. Pirali, M. Tudorie, F. P. Urena, and J.-J. Lopez Gonzalez, J. Quant. Spectrosc. Ra. 113 (2012) 1261.

30. P. D. Godfrey, D. McNaughton, and C. J. Evans, Chem. Phys. Lett. 580 (2013) 37.

31. D. Schmitz, V. A. Shubert, B. M. Giuliano, and M. Schnell, J. Chem. Phys. 141 (2014) 034304.

32. D. Schmitz, V. A. Shubert, T. Betz, and M. Schnell, Front. Chem. 3 (2015) 15.

33. V. A. Shubert, D. Schmitz, C. Medcraft, A. Krin, D. Patterson, J. M. Doyle, and M. Schnell, J. Chem. Phys. 142 (2015) 214201.

34. Z. Kisiel and A. C. Legon, J. Am. Chem. Soc. 100 (1978) 8166.

35. J. W. Bevan, A. C. Legon, S. O. Ljunggren, and P. J. Mjoberg, J. Am. Chem. Soc. 100 (1978) 8161.

36. J. R. Aviles Moreno, T. R. Huet, and E. Neeman, Microwave spectroscopy of monoterpenes of atmospheric interest: $\alpha$-pinene, $\beta$-pinene, and nopinone, in: 2014 International Symposium on Molecular Spectroscopy, volume 1, 2014.

37. C. J. Evans, private communication, 2015.

38. Z. Kisiel, O. Desyatnyk, E. Białkowska-Jaworska, and L. Pszczółkowski, Phys. Chem. Chem. Phys. 5 (2003) 820.

39. A. Choplin, Chem. Phys. Lett. 71 (1980) 503.

40. J. F. Chiang, R. Chiang, K. C. Lu, E.-M. Sung, and M. D. Harmony, J. Mol. Struct. 41 (1977) 67.

41. D. Schmitz, V. A. Shubert, T. Betz, and M. Schnell, J. Mol. Spectrosc. 280 (2012) 77.

42. C. M. Western, PGOPHER, a Program for Simulating Rotational Structure, 2013.

43. J. K. G. Watson, Mol. Phys. 15 (1968) 479.

44. H. M. Pickett, J. Mol. Spectrosc. 148 (1991) 371.

45. M. J. Frisch, G. W. Trucks, H. B. Schlegel, G. E. Scuseria, M. A. Robb, J. R. Cheeseman, G. Scalmani, V. Barone, B. Mennucci, G. A. Petersson, H. Nakatsuji, M. Caricato, X. Li, H. P. Hratchian, A. F. Izmaylov, J. Bloino, G. Zheng, J. L. Sonnenberg, M. Hada, M. Ehara, K. Toyota, R. Fukuda, J. Hasegawa, M. Ishida, T. Nakajima, Y. Honda, O. Kitao, H. Nakai, T. Vreven, J. A. Montgomery, J. E. Peralta, F. Ogliaro, M. Bearpark, J. J. Heyd, E. Brothers, K. N. Kudin, V. N. Staroverov, R. Kobayashi, J. Normand, K. Raghavachari, A. Rendell, J. C. Burant, S. S. Iyengar, J. Tomasi, M. Cossi, N. Rega, J. M. Millam, M. Klene, J. E. Knox, J. B. Cross, V. Bakken, C. Adamo, J. Jaramillo, R. Gomperts, R. E. Stratmann, O. Yazyev, 
A. J. Austin, R. Cammi, C. Pomelli, J. W. Ochterski, R. L. Martin, K. Morokuma, V. G. Zakrzewski, G. A. Voth, P. Salvador, J. J. Dannenberg, S. Dapprich, A. D. Daniels, Ö. Farkas, J. B. Foresman, J. V. Ortiz, J. Cioslowski, and D. J. Fox, Gaussian 09, 2009.

46. J. Kraitchman, Am. J. Phys. 21 (1953) 17.

47. Z. Kisiel, PROSPE. Programs for Rotational Spectroscopy. http://info.ifpan.edu.pl/ kisiel/ orospe.htm, 2015.

48. J. K. G. Watson, A. Roytburg, and W. Ulrich, J. Mol. Spectrosc. 196 (1999) 102.

49. S. M. Japar, T. J. Wallington, J. F. O. Richert, and J. C. Ball, Int. J. Chem. Kinet. 22 (1990) 1257.

50. T. H. Dunning Jr, J. Chem. Phys. 90 (1989) 1007.

51. U. Blukis, P. H. Kasai, and R. J. Myers, J. Chem. Phys. 38 (1963) 2753.

52. M. Hayashi and K. Kuwada, B. Chem. Soc. Jpn. 47 (1974) 3006.

Supplementary material: The online version of this article

(DOI: 10.1515/zpch-2015-0643) provides supplementary material for authorized users. 